\title{
Interaction-Induced Magnetization of the Two-Dimensional Electron Gas
}

\author{
Felix von Oppen, ${ }^{(1)}$ Denis Ullmo, ${ }^{(2)}$ and Harold U. Baranger ${ }^{(3)}$ \\ (1) Institut für Theoretische Physik, Zülpicher Str. 77, Universität zu Köln, 50937 Köln, Germany \\ ${ }^{(2)}$ Laboratoire de Physique Théorique et Modèles Statistiques (LPTMS), 91405 Orsay Cedex, France \\ ${ }^{(3)}$ Department of Physics, Duke University, Box 90305, Durham, NC 27708-0305
}

(Submitted to Phys. Rev. B, 7 January 2000)

\begin{abstract}
We consider the contribution of electron-electron interactions to the orbital magnetization of a two-dimensional electron gas, focusing on the ballistic limit in the regime of negligible Landaulevel spacing. This regime can be described by combining diagrammatic perturbation theory with semiclassical techniques. At sufficiently low temperatures, the interaction-induced magnetization overwhelms the Landau and Pauli contributions. Curiously, the interaction-induced magnetization is third-order in the (renormalized) Coulomb interaction. We give a simple interpretation of this effect in terms of classical paths using a renormalization argument: a polygon must have at least three sides in order to enclose area. To leading order in the renormalized interaction, the renormalization argument gives exactly the same result as the full treatment.
\end{abstract}

PACS: 73.20.Dx,71.10.Ca,03.65.Sq,05.30.Fk

\section{INTRODUCTION}

Within the independent-electron picture, the magnetic response of a bulk two-dimensional electron gas has two sources: Pauli paramagnetism originating from the electron spin and Landau diamagnetism originating from the orbital electronic motion. After studies of the contribution of superconducting fluctuations to the magnetic response of superconductors above $T_{c}$ [1,2], Aslamazov and Larkin [3] pointed out that electron-electron interactions make an analogous contribution to the magnetic response of normal-metal systems. While the fluctuation contribution is diamagnetic in superconductors, the Coulomb interaction gives a paramagnetic contribution to the susceptibility of normal metals; this difference is a direct consequence of the different signs of the effective interaction in the two cases.

In their seminal work, Aslamazov and Larkin [3] computed the interaction contribution to the susceptibility of three-dimensional metals and of layered systems at zero magnetic field. They found that the effect was particularly strong for layered systems. In view of the importance of the physics of the two-dimensional electron gas, the purpose of the present paper is to compute the interaction-induced magnetization of a strictly two-dimensional bulk system. We shall moreover go beyond the zero-field limit considered by Aslamazov and Larkin and compute the magnetization for arbitrary classically weak magnetic fields. We find that the interactioninduced magnetization generally dominates over the Landau and Pauli contributions at sufficiently low temperatures.

The relevant length scales of the problem are the thermal length $L_{T}=\hbar v_{F} /(2 \pi T)$, the magnetic length $L_{H}=$ $(\hbar / e B)^{1 / 2}$, the cyclotron radius $R_{c}=m_{e} v_{F} / e B$, and the elastic mean free path $\ell_{e l}$. Throughout this paper, we focus on the regime $L_{T} \ll \ell_{e l}$, which allows us to neglect the effects of impurity scattering. Moreover, we restrict ourselves to classically weak magnetic fields, defined by the condition $L_{T} \ll R_{c}$ (or equivalently $\hbar \omega_{c} \ll T$, where $\omega_{c}$ is the cyclotron frequency). Within a semiclassical approach, this implies that we can neglect the classical effects of the magnetic field on the trajectories and only need to consider the Aharonov-Bohm phases induced by the $B$ field. For classically weak magnetic fields, we can distinguish between two magnetic field regimes: The lowfield regime, considered by Aslamazov and Larkin, where $L_{T} \ll L_{H}$ and the high-field regime $L_{T} \gg L_{H}$. We present analytical results in both regimes and also show numerical results bridging these two regions. Most importantly, we present a simple renormalization argument based on classical paths which exactly reproduces the result of the complicated full treatment.

The magnetization of the two-dimensional electron gas has been studied experimentally in mesoscopic samples [4,5] and in the quantum-Hall regime [6, 9]. To the best of our knowledge, no experiments have been performed on bulk $2 \mathrm{~d}$ samples at classically weak magnetic fields. Such experiments would be a valuable test of our theoretical understanding of the interaction contribution to the magnetization.

This paper is organized as follows. In Sec. [1] we develop the semiclassical approach to the interactioninduced magnetization for $2 \mathrm{~d}$ bulk systems. In Sec. III we employ the general results derived in Sec. II to derive explicit expressions for the magnetization in the high- and low-field regimes. A curious feature of these results is that the interaction-induced susceptibility is third order in the (renormalized) interaction strength. In Sec. IV, we show how the renormalization group approach introduced in [10] allows one to give a simple semiclassical interpretation of this result. We conclude in Sec. V by comparing the interaction-induced susceptibility to the 
Landau and Pauli susceptibilities and discussing finitesize effects.

\section{THE SEMICLASSICAL APPROACH}

\section{A. Basics \\ Cooper-channel}

Calculating the interaction contribution to the magnetic response requires one to extend the high-density expansion (RPA) of the thermodynamic potential [11] by including interaction corrections from diagrams in the Cooper-channel. This was first done in the context of superconducting fluctuations and then applied to disordered normal metals 3,12 15. Such expansions usually give reliable results even beyond the high density limit, if the relevant sets of terms are properly resummed. The relevant Cooper-like diagrams are shown in Fig. 1. The screened Coulomb interaction (wavy lines) can be treated as local 13, 14: $U\left(\mathbf{r}-\mathbf{r}^{\prime}\right)=\lambda_{0} N(0)^{-1} \delta\left(\mathbf{r}-\mathbf{r}^{\prime}\right)$. Here, $N(0)=m_{e} /\left(\pi \hbar^{2}\right)$ denotes the full density of states and the bookkeeping index $\lambda_{0}=1$ identifies the order of perturbation. For a local interaction, the direct and exchange term are the same up to a factor of $(-2)$ coming from the spin sums and the different number of fermion loops. The straight lines in Fig. 1 represent finite-temperature Green functions of the non-interacting system. These take the form

$$
\mathcal{G}_{\mathbf{r}, \mathbf{r}^{\prime}}(\epsilon)=\theta(\epsilon) G_{\mathbf{r}, \mathbf{r}^{\prime}}^{R}\left(E_{F}+i \epsilon\right)+\theta(-\epsilon) G_{\mathbf{r}, \mathbf{r}^{\prime}}^{A}\left(E_{F}+i \epsilon\right)
$$

in terms of the retarded and advanced Green functions $G_{\mathbf{r}, \mathbf{r}^{\prime}}^{R}(E)=\left[G_{\mathbf{r}^{\prime}, \mathbf{r}}^{A}\left(E^{*}\right)\right]^{*}$.

The perturbation expansion for this interaction contribution $\Omega$ to the thermodynamic potential, which yields the magnetic response, can be formally expressed as 12,13

$$
\begin{aligned}
\Omega & =-\frac{1}{\beta} \sum_{n=1}^{\infty} \frac{\left(-\lambda_{0}\right)^{n}}{n} \sum_{\omega} \int d \mathbf{r}_{1} \ldots d \mathbf{r}_{n} \Sigma_{\omega}\left(\mathbf{r}_{1}, \mathbf{r}_{2}\right) \ldots \Sigma_{\omega}\left(\mathbf{r}_{n}, \mathbf{r}_{1}\right) \\
& =\frac{1}{\beta} \sum_{\omega} \operatorname{Tr}\left\{\ln \left[1+\lambda_{0} \hat{\Sigma}_{\omega}\right]\right\}
\end{aligned}
$$

Here, $\omega$ denotes the bosonic Matsubara frequencies $\omega=$ $2 \pi m / \beta$ ( $m$ is any positive or negative integer) with $\beta=1 / k_{B} T$. (We employ units such that $k_{B}=1$ in this paper.) The particle-particle propagator $\Sigma_{\omega}$ is expressed (in position representation) in terms of products of finitetemperature Green functions as 11]

$$
\Sigma_{\omega}\left(\mathbf{r}, \mathbf{r}^{\prime}\right)=\frac{1}{\beta N(0)} \sum_{\epsilon}^{E_{F}} \mathcal{G}_{\mathbf{r}, \mathbf{r}^{\prime}}(\epsilon) \mathcal{G}_{\mathbf{r}, \mathbf{r}^{\prime}}(\omega-\epsilon)
$$

where the sum runs over the fermionic Matsubara frequencies $\epsilon=(2 n+1) \pi / \beta$. The short-length (highfrequency) behavior is included in the screened interaction, thus requiring a cutoff of the frequency sums at the Fermi energy $E_{F}$ 13].

\section{Semiclassical Green function}

In view of the fact that the Fermi wavelength is the smallest length scale in the problem, our strategy will be to replace the free Green functions in the particle-particle propagator by their semiclassical approximations. Generally, the semiclassical approximation to the Green function $G_{\mathbf{r}, \mathbf{r}^{\prime}}\left(E_{F}+i \epsilon, B\right)$ is expressed as a sum over all classical paths from $\mathbf{r}$ to $\mathbf{r}^{\prime}$ at energy $E_{F}$ 16. For the bulk
$2 \mathrm{~d}$ electron gas, there is only a single such trajectory, namely the straight line connecting the two points. For $\epsilon=0$ and $B=0$ one therefore has

$$
G_{\mathbf{r}, \mathbf{r}^{\prime}}^{R}\left(E_{F}, B=0\right) \simeq \frac{1}{i \sqrt{2 \pi}} \frac{m_{e}}{\hbar^{2}} \frac{\exp \left[i S_{\mathbf{r}, \mathbf{r}^{\prime}}\left(E_{F}\right) / \hbar-i \pi / 4\right]}{\left(k_{F}\left|\mathbf{r}-\mathbf{r}^{\prime}\right|\right)^{1 / 2}}
$$

where $S_{\mathbf{r}, \mathbf{r}^{\prime}}\left(E_{F}\right)=\hbar k_{F}\left|\mathbf{r}-\mathbf{r}^{\prime}\right|$ is the classical action along the path. Moreover, since we assume the magnetic field is classically weak, the field affects the action along the path through

$$
S(B)=S(B=0)+\frac{e}{c} \int_{\mathbf{r}}^{\mathbf{r}^{\prime}} d \mathbf{x} \mathbf{A}(\mathbf{x})
$$

where the integral is along the unperturbed straight line path. Finally, it turns out that only small values of the imaginary part of the energy $\epsilon$ should be considered, so we can use the relation

$$
(\partial S / \partial E)=t
$$

where $t=\left|\mathbf{r}-\mathbf{r}^{\prime}\right| / v_{F}$ is the time of flight from $\mathbf{r}$ to $\mathbf{r}^{\prime}$. In this way, one obtains the semiclassical Green function for finite field and finite (imaginary) energy $\epsilon$ :

$$
G_{\mathbf{r}, \mathbf{r}^{\prime}}^{R}\left(E_{F}+i \epsilon, B\right)=G_{\mathbf{r}, \mathbf{r}^{\prime}}^{R}\left(E_{F}, B=0\right) \exp \left[\frac{i e}{\hbar c} \int_{\mathbf{r}}^{\mathbf{r}^{\prime}} d \mathbf{x} \mathbf{A}(\mathbf{x})\right] \exp \left[-\frac{|\epsilon|\left|\mathbf{r}-\mathbf{r}^{\prime}\right|}{\hbar v_{F}}\right] \text {. }
$$




$$
\hat{T}(\mathbf{R})=\exp \left(\frac{i}{\hbar} \mathbf{R} \cdot\left(\hat{\mathbf{p}}-\frac{2 e}{c} \mathbf{A}\right)\right)
$$

In the calculation of the thermodynamic potential Eq. (2) one should neglect all rapidly oscillating contributions in $\Sigma_{\omega}\left(\mathbf{r}, \mathbf{r}^{\prime}\right)$ as these will give a small contribution upon integration. Thus, in the particle-particle propagator $\Sigma$, it is necessary to pair advanced and retarded Green functions, and furthermore to pair each path in the semiclassical expression for $G^{R}$ with those in $G^{A}$ for which the dynamical phase factor cancels. The obvious case of pairing each path with itself is excluded because it yields no field dependence in $\Omega$ and hence zero magnetization. Thus one is led to consider pairs of time-reversed pathsfor these the dynamical phase cancels but the magnetic field part is multiplied by two. The pairing of $G^{R}$ with $G^{A}$ means concretely that one should keep only those terms in which $\epsilon$ and $\omega-\epsilon$ have opposite sign in the sum over Matsubara frequencies. Using the relation

$$
\sum_{\epsilon(\omega-\epsilon)<0} \exp [-|2 \epsilon-\omega| t / \hbar]=\frac{\exp [-|\omega| t / \hbar]}{\sinh (2 t \pi / \beta \hbar)},
$$

one obtains the final result for $\Sigma$

$$
\Sigma_{\omega}\left(\mathbf{r}, \mathbf{r}^{\prime}\right)=\Sigma_{\omega}^{(0)}\left(\left|\mathbf{r}-\mathbf{r}^{\prime}\right|\right) \exp \left\{\frac{2 i e}{c \hbar} \int_{\mathbf{r}}^{\mathbf{r}^{\prime}} d \mathbf{x} \mathbf{A}(\mathbf{x})\right\}
$$

where we have introduced the zero field limit of the particle-particle propagator

$$
\Sigma_{\omega}^{(0)}\left(\left|\mathbf{r}-\mathbf{r}^{\prime}\right|\right)=\frac{1}{4 \pi L_{T}\left|\mathbf{r}-\mathbf{r}^{\prime}\right|} \frac{\exp \left[-|m|\left|\mathbf{r}-\mathbf{r}^{\prime}\right| / L_{T}\right]}{\sinh \left(\left|\mathbf{r}-\mathbf{r}^{\prime}\right| / L_{T}\right)} .
$$

\section{B. Derivation of the eigenvalues}

The ladder-diagram contribution to the thermodynamic potential is expressed in Eq. (3) solely in terms of the eigenvalues of the operator $\Sigma_{\omega}\left(\mathbf{r}, \mathbf{r}^{\prime}\right)$. We therefore need to solve the eigenvalue equation

$$
\int d \mathbf{r}^{\prime} \Sigma_{\omega}\left(\mathbf{r}, \mathbf{r}^{\prime}\right) \psi_{n, l}\left(\mathbf{r}^{\prime}\right)=\sigma_{\omega}^{n, l} \psi_{n, l}(\mathbf{r})
$$

where $n$ and $l$ are quantum numbers.

Assuming from now on the symmetric gauge $\mathbf{A}=$ $\mathbf{B} \times \mathbf{r} / 2$, Eq. (10) reads

$$
\Sigma_{\omega}\left(\mathbf{r}, \mathbf{r}^{\prime}\right)=\Sigma_{\omega}^{(0)}\left(\left|\mathbf{r}-\mathbf{r}^{\prime}\right|\right) \exp \left\{\frac{2 i e}{c \hbar} \frac{B}{2}\left(\mathbf{r} \times \mathbf{r}^{\prime}\right)\right\} .
$$

It can be easily checked that any operator of the form Eq. (13) commutes with any element of the magnetic translation group and therefore with its generators

$$
\begin{aligned}
& \hat{\Pi}_{x}=\left(\hat{p}_{x}-(2 e / c) A_{x}\right) \\
& \hat{\Pi}_{y}=\left(\hat{p}_{y}-(2 e / c) A_{y}\right) .
\end{aligned}
$$

Noting that, first, $\Sigma_{\omega}\left(\mathbf{r}, \mathbf{r}^{\prime}\right)$ is invariant under rotation and, second, the Landau Hamiltonian for a particle of charge $(-2 e)$ can be written as

$$
\hat{H}_{L}=\left(\hat{\mathbf{p}}+\frac{2 e}{c} \mathbf{A}\right)^{2}=\hat{\Pi}_{x}^{2}+\hat{\Pi}_{y}^{2}+\frac{4 e}{c \hbar^{2}} \hat{J}_{z}
$$

we see that $\Sigma_{\omega}\left(\mathbf{r}, \mathbf{r}^{\prime}\right)$ is diagonal in the basis $\left\{\psi_{n, l}\right\}$ of the eigenvectors of $\hat{H}_{L}$ and $\hat{J}_{z}$, where $n$ and $l$ are the Landau level and angular momentum quantum numbers, respectively. For $l=0$ the Landau level wavefunction (for a particle of charge $-2 e$ ) has the well-known form

$$
\psi_{n, 0}(\mathbf{r})=\exp \left\{-\frac{|\mathbf{r}|^{2}}{2 L_{H}^{2}}\right\} L_{n}\left(\frac{|\mathbf{r}|^{2}}{L_{H}^{2}}\right)
$$

with $L_{n}$ the Laguerre polynomial and $L_{H}=(\hbar / e B)^{1 / 2}$.

Finally, an important property of the $\hat{T}(\mathbf{R})$ is that they commute with both $\hat{H}_{L}$ and $\Sigma_{\omega}\left(\mathbf{r}, \mathbf{r}^{\prime}\right)$ but not with $J_{z}$. Since, moreover, within a Landau level there is no stable subspace for all the $\hat{T}(\mathbf{R})$, the eigenvalues $\sigma_{\omega}^{n, l}$ cannot depend on the angular momentum quantum number $l$. At $\mathbf{r}=0$, Eq. (12), then, reads

$$
\sigma_{\omega}^{n, l}=\sigma_{\omega}^{n, 0}=\frac{1}{\psi_{n, 0}(0)} \int d \mathbf{r} \Sigma_{\omega}^{(0)}(|\mathbf{r}|) \psi_{n, 0}(\mathbf{r})
$$

Using the explicit expressions for $\Sigma_{\omega}^{(0)}(\mathbf{r})$ [Eq. (11)] and $\psi_{n, 0}$, we finally obtain for the eigenvalues

$$
\begin{aligned}
\sigma_{\omega}^{n, l} & =\sigma_{\omega}^{n, 0} \\
& =\frac{1}{2} \int_{x_{\min }}^{\infty} d x \frac{\exp \{-|m| x\}}{\sinh x} \exp \left\{-x^{2} / 2 \alpha^{2}\right\} L_{n}\left(x^{2} / \alpha^{2}\right)
\end{aligned}
$$

where $\alpha=L_{H} / L_{T}$ is the essential dimensionless parameter. It is important to keep in mind that the screened interaction already implicitly takes into account the effect of the interaction on scales shorter than the Fermi wavelength, so the integral over $x$ should be cut off for small $x$ at approximately $x_{\min }=1 /\left(k_{F} L_{T}\right)$. 


\section{Reordering of the sum}

The interaction contribution to the thermodynamic potential is given in terms of the eigenvalues $\sigma_{\omega}^{n}$ by

$$
\Omega=\frac{2 B A}{\phi_{0}} \frac{1}{\beta} \sum_{\omega} \sum_{n=0}^{\infty} \ln \left(1+\lambda_{0} \sigma_{\omega}^{n}\right)
$$

Here we have already taken proper account of the degeneracy of the eigenvalues by the prefactor $2 B A / \phi_{0}$. The magnetization per unit area now follows by differentiation with respect to $B$,

$$
M=-\frac{2}{\phi_{0} \beta} \sum_{\omega} \sum_{n=0}^{\infty}\left\{\ln \left(1+\lambda_{0} \sigma_{\omega}^{n}\right)+\frac{B \lambda_{0}}{1+\lambda_{0} \sigma_{\omega}^{n}} \frac{\partial \sigma_{\omega}^{n}}{\partial B}\right\}
$$

When done naively, the sum over the quantum number in this expression diverges. We assume that this is associated with the inadequate treatment of the interaction at short distances. We expect that when working with the full screened interaction, the contribution of large quantum numbers is appropriately suppressed. Hence, we reorder the sum in such a way that the sum becomes convergent and the eigenvalues with sufficiently large $n$ do not contribute appreciably to the sum. This philosophy is completely analogous to the approach taken in the work on the fluctuation contribution to the diamagnetic susceptibility in superconductors above $T_{c}$ [1]:2]. In fact, our reordering closely follows the reordering proposed by Payne and Lee [1].

In a first step, we compute

$$
B \frac{\partial \sigma_{\omega}^{n}}{\partial B}=2 \pi \int_{0}^{\infty} d \rho \rho \Sigma_{\omega}^{(0)}(\rho) \exp \left\{-\frac{e B}{2 \hbar} \rho^{2}\right\} \frac{e B}{\hbar} \rho^{2}\left[L_{n}^{\prime}\left(e B \rho^{2} / \hbar\right)-\frac{1}{2} L_{n}\left(e B \rho^{2} / \hbar\right)\right]
$$

To simplify this expression, we use the recursion relations for Laguerre polynomials

$$
\begin{aligned}
& x L_{n}^{\prime}(x)=n L_{n}(x)-n L_{n-1}(x) \\
& x L_{n}^{\prime}(x)=(n+1) L_{n+1}(x)-(n+1-x) L_{n}(x)
\end{aligned}
$$

and obtain

$$
2 B \frac{\partial \sigma_{\omega}^{n}}{\partial B}=(n+1)\left[\sigma_{\omega}^{n+1}-\sigma_{\omega}^{n}\right]+n\left[\sigma_{\omega}^{n}-\sigma_{\omega}^{n-1}\right]
$$

We can also rearrange

$$
\begin{aligned}
\sum_{n=0}^{\infty}(n+1) \ln \frac{f_{n+1}}{f_{n}} & =\sum_{n=0}^{\infty} n \ln f_{n}-\sum_{n=0}^{\infty}(n+1) \ln f_{n} \\
& =-\sum_{n=0}^{\infty} \ln f_{n} .
\end{aligned}
$$

Using these expressions, we have for the magnetization

$$
M=\frac{1}{\phi_{0}} \frac{1}{\beta} \sum_{\omega} \sum_{n=0}^{\infty}(n+1)\left\{2 \ln \frac{1+\lambda_{0} \sigma_{\omega}^{n+1}}{1+\lambda_{0} \sigma_{\omega}^{n}}-\frac{\lambda_{0}\left[\sigma_{\omega}^{n+1}-\sigma_{\omega}^{n}\right]}{1+\lambda_{0} \sigma_{\omega}^{n}}-\frac{\lambda_{0}\left[\sigma_{\omega}^{n+1}-\sigma_{\omega}^{n}\right]}{1+\lambda_{0} \sigma_{\omega}^{n+1}}\right\} .
$$

In terms of the notation

$$
X_{\omega}^{n}=\frac{\lambda_{0}\left[\sigma_{\omega}^{n+1}-\sigma_{\omega}^{n}\right]}{1+\lambda_{0} \sigma_{\omega}^{n}}
$$

we have

$$
M=\frac{1}{\phi_{0}} \frac{1}{\beta} \sum_{\omega} \sum_{n=0}^{\infty}(n+1)\left\{2 \ln \left(1+X_{\omega}^{n}\right)-X_{\omega}^{n}-\frac{X_{\omega}^{n}}{1+X_{\omega}^{n}}\right\} .
$$

For all cases considered below, $X_{\omega}^{n} \ll 1$ so that

$$
M \simeq-\frac{1}{\phi_{0}} \frac{1}{\beta} \sum_{\omega} \sum_{n=0}^{\infty} \frac{(n+1)}{3}\left[X_{\omega}^{n}\right]^{3} .
$$


This expression will be our starting point for computing the magnetization and the susceptibility. Referring back to the definition of $X_{\omega}^{n}$ in Eq. (27) above, we see that all contributions to the susceptibility are at least third-order in the interaction $\lambda_{0}$.

\section{MAGNETIC SUSCEPTIBILITY}

Using the general results of the last section, we now find expressions for the susceptibility in two limits- small and large magnetic field- and then evaluate the suscep- tibility in the intermediate regime numerically. Before considering the various regimes, it is useful to note that $X_{\omega}^{n}$, as defined in Eq. (27), consists of two factors with noticeably different behavior. On the one hand, because the integrand in Eq. (18) behaves as $1 / x$ at small $x$, both $\sigma_{\omega}^{n}$ and $\lambda_{0} /\left(1+\lambda_{0} \sigma_{\omega}^{n}\right)$ are dominated by a logarithmic singularity at zero and so have little magnetic field dependence. On the other hand, using the relation $L_{n}(x)-L_{n-1}(x)=-x L_{n-1}^{1}(x) / n\left(L_{n}^{1}\right.$ is a generalized Laguerre polynomial), we can rewrite $\Delta \sigma_{\omega}^{n} \equiv \sigma_{\omega}^{n+1}-\sigma_{\omega}^{n}$ as

$$
\Delta \sigma_{\omega}^{n}=-\frac{1}{(n+1)} \frac{1}{2 \alpha^{2}} \int_{0}^{\infty} d x x^{2} \frac{e^{-|m| x}}{\sinh x} \exp \left\{-x^{2} / 2 \alpha^{2}\right\} L_{n}^{1}\left(x^{2} / \alpha^{2}\right)
$$

Here the $x$ integration is well behaved at small $x$, and so the lower limit $x_{\min }$ can be replaced by zero.

\section{A. Small-magnetic-field (high-temperature) limit}

The small-magnetic field, or equivalently hightemperature, limit is defined by $\alpha \gg 1$. The factor $e^{-|m| x} / \sinh x$ provides an upper cutoff at $\min \left(1,|m|^{-1}\right)$ in the integrals Eqs. (18) and (30). In addition, $x^{2} / \alpha^{2}$ is much smaller than one in the entire range of integration. We can therefore use the asymptotic expression [17]

$e^{-x / 2} L_{n}^{\alpha}(x) \simeq \frac{\Gamma(\alpha+n+1)}{n !}(\nu x / 4)^{-\alpha / 2} J_{\alpha}\left([\nu x]^{1 / 2}\right)$,

valid in the range $0 \leq x \leq n^{1 / 3}\left[J_{\alpha}(x)\right.$ denotes the Bessel functions, $\Gamma(n)$ the Gamma function, and $\nu=4 n+2 \alpha+2]$ and obtain

$$
\begin{aligned}
\sigma_{\omega}^{n} & \simeq \frac{1}{2} \int_{x_{\min }}^{\infty} d x \frac{\exp (-|m| x)}{\sinh x} J_{0}(2 \sqrt{n+1 / 2} x / \alpha) \\
\Delta \sigma_{\omega}^{n} & \simeq-\frac{1}{\sqrt{n+1}} \frac{1}{2 \alpha} \int_{0}^{\infty} d x x \frac{\exp (-|m| x)}{\sinh x} J_{1}\left(\frac{2 \sqrt{n+1} x}{\alpha}\right)
\end{aligned}
$$

For $n \ll n_{0}=\alpha^{2} \cdot \max (1,|m|)$, Eqs. (32) and (33) yield 18

$$
\sigma_{\omega}^{n} \simeq \frac{1}{2} \int_{x_{\min }}^{\min \left(1,|m|^{-1}\right)} \frac{d x}{x}=\frac{1}{2} \ln \left(k_{F} L_{T} / \max \{1,|m|\}\right)
$$

$$
\Delta \sigma_{\omega}^{n} \simeq-\frac{1}{2 \alpha^{2}} \int_{0}^{\infty} d x x^{2} \frac{e^{-|m| x}}{\sinh x}
$$

up to constants of order one, and so the $n$ dependence can be neglected. For $n>n_{0}$, both $\Delta \sigma_{\omega}^{n}$ and $\sigma_{\omega}^{n}$ depend on $n$. However, for $\sigma_{\omega}^{n}$ the dependence is only logarithmic, since it merely amounts to replacing the upper bound of the integral by $\alpha / \sqrt{n}$. Hence the dominant $n$ dependence of $X_{\omega}^{n}$ comes from $\Delta \sigma_{\omega}^{n}$.

From these results for the eigenvalues, the magnetization [Eq. (29)] to lowest order in the small parameter $\ln ^{-1}\left(k_{F} L_{T}\right)$ is

$$
M=\frac{1}{3 \phi_{0}} \frac{1}{\beta} \sum_{\omega} \frac{1}{\alpha^{3} \ln ^{3}\left(k_{F} L_{T}\right)} \sum_{n=1}^{\infty} \frac{1}{\sqrt{n}}\left\{\int_{0}^{\infty} d x \frac{x \exp (-|m| x)}{\sinh x} J_{1}(2 \sqrt{n} x / \alpha)\right\}^{3} .
$$

The sum over $n$ converges only slowly and of order $n_{0}$ terms contribute. In view of the fact that $n$ is multiplied by $x^{2} / \alpha^{2}$ in the argument of the Bessel function, we can replace the sum over $n$ by an integral. This yields the final expression

$$
M=\frac{C_{T}}{\pi \ln ^{3}\left(k_{F} L_{T}\right)}\left(k_{F} L_{T}\right)\left|\chi_{\text {Landau }}\right| B
$$

where $\left|\chi_{\text {Landau }}\right|=e^{2} / 12 \pi m c^{2}$ and $C_{T}$ is given by

$$
C_{T}=\sum_{r=-\infty}^{\infty} \int_{0}^{\infty} \frac{d n}{n^{2}} f_{\omega}^{3}(n) \simeq 0.97
$$

where we define

$$
f_{\omega}(n) \equiv \sqrt{n} \int_{0}^{\infty} d x \frac{x \exp (-|m| x)}{\sinh x} J_{1}(2 \sqrt{n} x) .
$$


We see that the interaction-induced contribution to the magnetization will generally be larger than the Landau magnetization due to the large factor $k_{F} L_{T}$ [3]. The factor $1 / \ln \left(k_{F} L_{T}\right)$ must be interpreted as a renormalized interaction strength in the Cooper channel [3,13]. It is interesting that the interaction contribution to the susceptibility is third order in this renormalized interaction strength. This unusual state of affairs can easily be understood by considering the classical paths involved, as we shall discuss in Section IV.

The magnetic susceptibility $\chi$ obtained above is at zero field and to lowest order in the renormalized interaction strength which is proportional to $1 / \ln \left(k_{F} L_{T}\right)$. It is possible to derive, with a similar approach, an expression for $\chi(0)$ without expanding in the renomalized interaction strength. This is done in Appendix A; one obtains

$$
\frac{\chi(0)}{\left|\chi_{\text {Landau }}\right|}=\frac{3}{\pi}\left(k_{F} L_{T}\right) \sum_{\omega} \int_{0}^{\infty} \frac{d \xi}{\xi^{2}} \lambda_{\omega}^{3}(\xi) f_{\omega}^{3}(\xi)
$$

where we have introduced

$$
\begin{aligned}
\lambda_{\omega}(\xi) & =\frac{\lambda_{0}}{2+\lambda_{0}\left(2 \sigma^{0}(T)-g_{\omega}(\xi)\right)} \\
g_{\omega}(x) & =\int_{0}^{\xi} \frac{f_{\omega}\left(\xi^{\prime}\right)}{\xi^{\prime}} d \xi^{\prime} \\
2 \sigma^{0} & \equiv 2 \sigma_{\omega}^{n}(B=0)=\int_{x_{\min }}^{\infty} d x \frac{e^{-|m| x}}{\sinh x} \\
& \simeq \ln \left(k_{F} L_{T} / \max (1,|m|)\right) .
\end{aligned}
$$

\section{B. Large-magnetic-field (low-temperature) limit}

In the high-magnetic-field, or equivalently the lowtemperature, limit defined by $\alpha \ll 1$, the factor $\exp \left\{-x^{2} / 2 \alpha^{2}\right\} L_{n}\left(x^{2} / \alpha^{2}\right)$ always cuts off the integral in Eq. (18) at $x \ll 1$ so that we can approximate $\sinh x \simeq x$. Hence, we find

$$
\begin{aligned}
\sigma_{\omega}^{n} & \simeq \frac{1}{2} \int_{x_{\min }}^{\min \left[|m|^{-1}, \alpha / \sqrt{n}\right]} \frac{d x}{x} \\
& =\frac{1}{2} \min \left[\ln \left(k_{F} L_{H} / \sqrt{n}\right), \ln \left(k_{F} L_{T} /|m|\right)\right] \\
\Delta \sigma_{\omega}^{n} \simeq- & -\frac{1}{2(n+1)} \int_{0}^{\infty} d y y e^{-\alpha|m| y-y^{2} / 2} L_{n}^{1}\left(y^{2}\right) .
\end{aligned}
$$

In this case, the sum over $n$ converges rapidly (faster than $1 / n^{2}$ ), but typically about $1 / \alpha$ terms contribute to the Matsubara sum. Neglecting again the logarithmic dependence of $\sigma_{\omega}^{n}$ on $n$ and $|m|$, we can make progress by noting that the sum over $m$ can be turned into an integral. This yields the high-field result

$$
M=\frac{C_{H}}{\pi \ln ^{3}\left(k_{F} L_{H}\right)}\left(k_{F} L_{H}\right)\left|\chi_{\text {Landau }}\right| B
$$

where the constant $C_{H}$ is

$$
C_{H}=\int_{-\infty}^{\infty} d m \sum_{n=0}^{\infty} \frac{\left(F_{n}(m)\right)^{3}}{(n+1)^{2}} \simeq 0.74
$$

with

$$
F_{n}(m)=\int_{0}^{\infty} d y y e^{-|m| y-y^{2} / 2} L_{n}^{1}\left(y^{2}\right) .
$$

The principal difference between the results for high and low fields is thus the replacement of the thermal length $L_{T}$ by the magnetic length $L_{H}$.

\section{Intermediate range}

When $\alpha$ is neither much smaller nor much larger than one, it is not possible to obtain a simple expression for the magnetic response. In this regime, we have performed a numerical integration of Eq. (18) to compute the eigenvalues $\sigma_{\omega}^{n}$ as well as their derivatives with respect to $B$. The magnetic susceptibility is then obtained through the field derivative of Eq. (28),

$$
\chi=\frac{-1}{\beta \phi_{0}} \sum_{\omega} \sum_{n} n \frac{\left(X_{\omega}^{n-1}\right)^{2} d X_{\omega}^{n-1} / d B}{\left(1+X_{\omega}^{n-1}\right)^{2}},
$$

by direct summation over the eigenvalue index and the bosonic Matsubara frequencies.

Fig. 2 2 shows the resulting $\chi /\left|\chi_{\text {Landau }}\right|$ as a function of magnetic field $b=\alpha^{-2}=\left(2 \pi L_{T}^{2} / \phi_{0}\right) B$ for fixed values of the temperature. The cross-over between the low and high field regimes is clearly seen. As $B$ increases, $\chi$ has a slight maximum around $L_{H}=L_{B}$ which arises from the competition between the increased field sensitivity of large triangles and the thermal suppression of long sides. In the large-field regime, the numerical result is in reasonable agreement with the value obtained from the asymptotic expression (46): for $L_{T}=10 L_{H}$ and $k_{F} L_{H}=64, \chi /\left|\chi_{\text {Landau }}\right| \approx 0.28$ numerically and 0.18 analytically.

Fig. 3 shows the temperature dependence of $\chi$ at fixed $L_{H} / L_{T}$ in the low-field regime. The $\left(k_{F} L_{T}\right) / \ln ^{3}\left(k_{F} L_{T}\right)$ behavior is apparent, particularly in the inset. Again, the numerical result agrees nicely with the asymptotic result: at the low temperature $T / E_{F}=10^{-3}$, Eq. (37) yields $\chi /\left|\chi_{\text {Landau }}\right| \approx 0.51$ while our numerical result is 0.52 . 


\section{SEMICLASSICAL INTERPRETATION}

We have seen above that the magnetic response has a rather peculiar property: it is third-order in the renormalized coupling constant $\tilde{\lambda}=2 \ln ^{-1}\left(k_{F} L_{T, H}\right)$, both in the low and high field regimes. Within the approach used up to now, it is difficult to understand the physical origin of this behavior. In this section, we show that an approach in terms of classical paths provides a natural understanding of this fact. Considering for instance the low field regime (the argument can be transposed to high fields with no essential difficulty), we shall moreover re- cover precisely the expression Eq. 37. in a much simpler way.

From the expression Eq. (10) for the particle-particle propagator, it is clear that the interaction contribution to the thermodynamic potential in Eq. (2) can be written as a sum over closed polygonal paths, where each vertex is associated with an interaction event and the magnetic field enters only via the Aharonov-Bohm factor associated with the magnetic flux enclosed by the polygon. Performing the sum over bosonic Matsubara frequencies in Eq. (2) and grouping the field dependent terms together, one finds

$$
\begin{gathered}
\Omega=\sum_{n=1}^{\infty} \Omega^{(n)} \\
\Omega^{(n)}=-\frac{1}{\beta} \frac{\left(-\lambda_{0}\right)^{n}}{n} \int d \mathbf{r}_{1} \ldots d \mathbf{r}_{n} \tilde{\Sigma}\left(\mathbf{r}_{1}, \mathbf{r}_{2}\right) \ldots \tilde{\Sigma}\left(\mathbf{r}_{n}, \mathbf{r}_{1}\right) \operatorname{coth}\left(\frac{L_{\text {tot }}\left(\mathbf{r}_{1}, \ldots, \mathbf{r}_{n}\right)}{2 L_{T}}\right) \cos \left(\frac{4 \pi A_{\text {tot }}\left(\mathbf{r}_{1}, \ldots, \mathbf{r}_{n}\right) B}{\phi_{0}}\right)
\end{gathered}
$$

where $\tilde{\Sigma}\left(\mathbf{r}, \mathbf{r}^{\prime}\right)$ is the particle-particle propagator for $B=0$ and $\omega=0, L_{\text {tot }}$ is the total length, and $A_{\text {tot }}$ the area enclosed by the polygon.

As it stands, Eq. (50) is of little practical use because the series in $n$ is strongly divergent (the term of order $n$ is typically larger than the one of order $n-1)$. It is, however, possible to apply a simple renormalization-group argument, introduced in [10] and discussed in more detail in [22]. Indeed, as we already stressed when deriving the eigenvalues of the particle-particle propagator, $\tilde{\Sigma}\left(\mathbf{r}, \mathbf{r}^{\prime}\right)$ must be cutoff at $\Lambda_{0}=1 / k_{F}$ because the use of the screened interaction assumes that all high-momentum degrees of freedom have already been integrated out [13]. We therefore have

$$
\begin{aligned}
\tilde{\Sigma}\left(\mathbf{r}, \mathbf{r}^{\prime} ; \Lambda_{0}\right) & =\frac{1}{4 \pi L_{T}\left|\mathbf{r}-\mathbf{r}^{\prime}\right| \sinh \left(\left|\mathbf{r}-\mathbf{r}^{\prime}\right| / L_{T}\right)} & \text { for }\left|\mathbf{r}-\mathbf{r}^{\prime}\right|>\Lambda_{0} \\
& =0 & \text { for }\left|\mathbf{r}-\mathbf{r}^{\prime}\right|<\Lambda_{0}
\end{aligned}
$$

Refs. 10,22 show that if a new length scale $\Lambda>\Lambda_{0}$ much smaller that any other characteristic length scale of the problem $\left(L_{T}\right.$ or $\left.L_{H}\right)$ is introduced, one can replace $\Lambda_{0}$ by $\Lambda$ in Eq. (52) provided the "bare" coupling constant $\lambda_{0}$ in Eq. (50) is replaced by the renormalized one

$$
\lambda_{R G}(\Lambda)=\frac{\lambda_{0}}{1+\left(\lambda_{0} / 2\right) \ln \left(\Lambda / \Lambda_{0}\right)} .
$$

For the leading behavior in $\ln ^{-1}\left(k_{F} L_{T}\right)$, we can assume $\Lambda=\epsilon L_{T}$ with $\epsilon \ll 1$ but assumed fixed as $\ln \left(k_{F} L_{T}\right)$ goes to infinity. In that case $\lambda_{R G}(\Lambda)=2 \ln ^{-1}\left(k_{F} L_{T}\right)+O\left(\ln ^{-2}\left(k_{F} L_{T}\right)\right)$ is small, and Eq. (50) becomes a genuine perturbative expansion whose leading behavior is given by the first non-vanishing term. Clearly, $\Omega^{(1)}=0$, and $\Omega^{(2)}$ is independent of the magnetic field and so does not contribute to the magnetic response. Therefore, the leading behavior is given

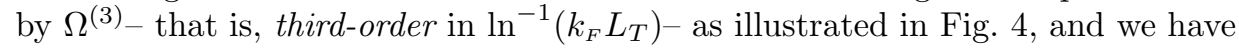

$$
\chi(B=0)=\frac{1}{3 \beta}\left(\frac{2}{\ln \left(k_{F} L_{T}\right)}\right)^{3} \int_{L_{2}, L_{3}, L_{23}>\Lambda} d \mathbf{r}_{2} d \mathbf{r}_{3} \tilde{\Sigma}\left(\mathbf{0}, \mathbf{r}_{2}\right) \tilde{\Sigma}\left(\mathbf{r}_{2}, \mathbf{r}_{3}\right) \tilde{\Sigma}\left(\mathbf{r}_{2}, \mathbf{0}\right) \operatorname{coth}\left(\frac{L_{\text {tot }}}{2 L_{T}}\right)^{\left(\frac{4 \pi A_{\text {tot }}}{\phi_{0}}\right)^{2}}
$$

$\left(L_{2}=\left|\mathbf{r}_{2}\right|, L_{3}=\left|\mathbf{r}_{3}\right|, L_{23}=\left|\mathbf{r}_{3}-\mathbf{r}_{2}\right|\right)$. Expressing all distances in the integral in units of $L_{T}$ gives

$$
\chi(B=0)=\frac{C_{R G}}{\pi \ln ^{3}\left(k_{F} L_{T}\right)}\left(k_{F} L_{T}\right)\left|\chi_{\text {Landau }}\right|
$$

with the constant $C_{R G}$ given by

$$
C_{R G}=\frac{1}{\pi^{2}} \int d \mathbf{r}_{2} d \mathbf{r}_{3} \frac{1}{L_{2} L_{23} L_{3}} \frac{A_{\mathrm{tot}}^{2} \operatorname{coth}\left(L_{\mathrm{tot}} / 2\right)}{\sinh L_{2} \sinh L_{23} \sinh L_{3}}
$$

Because of the factor $A_{\text {tot }}^{2}$ in the numerator, the inte- 
grand here is regular and the cutoff can be taken to zero. By considering the limit $\lambda_{0} \ll \ln \left(k_{F} L_{T}\right) \ll 1$ (instead of $\lambda_{0}=1$ ) where both the standard and renormalization group approaches are accurate, it can be shown that $C_{R G}=C_{T}$, so that Eq. (55) is strictly equivalent to Eq. (37). This approach shows clearly that the third power of the coupling constant arises because only trajectories with three or more vertices enclose flux.

\section{DISCUSSION AND SUMMARY}

In this paper, we study the interaction contribution to the magnetization of a two-dimensional electron gas in the limit $L_{T} \ll R_{c}$, $\ell_{\mathrm{el}}$. We find that this interactioninduced contribution is paramagnetic for the repulsive Coulomb interaction and dominates over the Landau diamagnetism at small enough temperatures and fields [3]. The Pauli paramagnetism is even smaller than Landau diamagnetism in GaAs/AlGaAs heterostructures because of the small effective mass and the reduction in the $g$ factor. It appears from the quantitative answer that one needs to go to rather low temperatures before the interaction becomes truly larger than the Landau susceptibility (cf. Fig. 3). Still, such temperatures are possible in two-dimensional electron gas systems. It should be possible to distinguish the interaction contribution by way of either its temperature dependence (since the Landau susceptibility is $T$ independent) or its dependence on $k_{F}$ in a gated structure.

We find that the leading contribution to the magnetization is third-order in the renormalized Coulomb interaction. This can be given a natural semiclassical interpretation in terms of the classical-path picture for the thermodynamic potential: a polygon must have at least three sides in order to enclose area. Moreover, the much simpler classical-path approach gives exactly the same answer as the eigenvalue calculation. Higher-thanthird order contributions (in the bare coupling constant) predominantly lead to an substantial downward renormalization of the coupling constant. This picture has been made precise in the present paper by means of a renormalization-group approach.

In the low-field (high-temperature) limit $L_{T} \ll L_{H}$, the temperature dependence of the susceptibility is $1 / T \ln ^{3} T$ : this comes from the thermal length $L_{T}$ which dominates here because only trajectories shorter than $L_{T}$ make significant contributions to the Green functions. At the lowest temperatures, this behavior is cut off by a finite magnetic field once $L_{H}<L_{T}$. In the high-field (low-temperature) limit $L_{T} \gg L_{H}$, the susceptibility is no longer temperature dependent. The Green function is still dominated by trajectories shorter than $L_{T}$, but now trajectories enclosing more area than $L_{H}^{2}$ contribute with random signs due to the Aharonov-Bohm phases. Hence, in this case, the relevant cutoff length is $L_{H}$.
So far, we ignored dephasing due to inelastic scattering. At low temperatures, this should be mostly due to electron-electron scattering. We expect however that dephasing will not significantly affect our results. Within the semiclassical approach employed in this paper, dephasing suppresses the contribution of trajectories longer than the dephasing length $L_{\phi}$. For a clean Fermi liquid such as discussed here, one expects $L_{\phi} \sim 1 / T^{2}$. Thus, at sufficiently low temperatures the dephasing length should always be longer than the thermal length $L_{T} \sim 1 / T$. Correspondingly, the suppression of trajectories due to thermal smearing should always set in before the suppression due to dephasing.

It is interesting to compare the present results with the contributions to the susceptibility of (chaotic) mesoscopic samples of linear size $L$ within the independent-electron approximation 19 21

$$
\chi \sim\left|\chi_{\text {Landau }}\right|
$$

and due to interactions 10

$$
\chi \sim \frac{\left(k_{F} L\right)}{\ln \left(k_{F} L\right)}\left|\chi_{\text {Landau }}\right|,
$$

where we have taken $L_{T} \sim L, T>\Delta$ ( $\Delta$ is the level spacing), and $L_{H} \gg L$. In contrast to the bulk results derived in this paper, these expressions are zeroth or first order in the renormalized interaction constant. These contributions exist for mesoscopic samples because, in finitesize systems, flux-enclosing trajectories are produced by scattering from the geometric boundaries of the system. Nevertheless, apart from the different order in the renormalized interaction, the finite-size result due to interactions is qualitatively the same as the bulk result derived here.

Orbital magnetism in mesoscopic samples has been a controversial issue over the last decade, both for ballistic and diffusive structures, ring and dot geometries [4, 23, 26]. In particular, the fact that the measured values are apparently substantially larger than the theoretical results has attracted a lot of attention. In order to benchmark the theory in a simpler system, we think the magnetization of a clean two-dimensional electron gas should be measured and that this would provide valuable information in addressing the "persistent current problem" in rings and dots.

\section{ACKNOWLEDGMENTS}

We thank Rodolfo Jalabert and Klaus Richter for valuable discussions. The LPTMS is "Unité de recherche de l'Université Paris 11 associée au C.N.R.S.". FvO was partly supported by SFB 341 (Köln-Aachen-Jülich). 


\section{APPENDIX A:}

In this appendix, we derive an expression for the susceptibility at zero field, Eq. (40), which does not involve expanding in the renormalized coupling constant. Starting from the magnetization Eq. (29), the essential ingredient needed is expressions for the eigenvalues. One can check that both the Bessel approximations Eqs. (32)(33) and the replacement of the discrete sum over Landau level index $n$ by an integral only yield corrections of order $B^{2}$ to the magnetic susceptibility. Therefore, as long as we are only interested in the susceptibility at $B=0$, we can make the change of variables $n \rightarrow \xi=b n$ where $b=\alpha^{-2}=\left(2 \pi L_{T}^{2} / \phi_{0}\right) B$ is proportional to the magnetic field. We can thus write

$$
2 \Delta \sigma_{\omega}^{n-1}=-b f_{\omega}(\xi) / \xi
$$

where $f_{\omega}(\xi)$ is defined by Eq. (39). In the same way, taking the derivative of Eq. (32) with respect to $b$ yields

$$
2 \frac{d \sigma_{\omega}^{n-1}}{d b}=-\frac{1}{b} f((n-1 / 2) b) \simeq-\frac{1}{b}[f(\xi)+O(b)] .
$$

Thus, using the notation of Eqs. (42) and (43),

$$
2 \sigma_{\omega}^{n-1}=2 \sigma^{0}-g((n-1 / 2) b) \simeq 2 \sigma^{0}-g(\xi)+O(b) .
$$

From these expressions for the eigenvalues, we obtain

$$
\begin{aligned}
X_{\omega}^{n-1}=X_{\omega}(\xi, b) & =-b \frac{\lambda_{0} f_{\omega}(\xi) / \xi}{2+\lambda_{0}\left(2 \sigma^{0}-g(\xi)\right)}+O\left(b^{2}\right) \\
& =-b \lambda_{\omega}(\xi) f_{\omega}(\xi) / \xi+O\left(b^{2}\right) .
\end{aligned}
$$

Note that it is necessary to compensate the factor $b^{-2}$ originating from the term $n d n$ when changing variables from $n$ to $\xi$. Using the above expression, one can therefore write the magnetization up to corrections of order $b^{2}$,

$$
M=-\frac{1}{3 \phi_{0} \beta} \sum_{\omega} \int \frac{\xi d \xi}{b^{2}} X_{\omega}(\xi, b)^{3}+O\left(b^{2}\right)
$$

which immediately gives Eq. (40).

[1] P.A. Lee and M.G. Payne, Phys. Rev. B 5, 923 (1972).

[2] J. Kurkijärvi, V. Ambegaokar, and G. Eilenberger, Phys. Rev. B 5, 868 (1972).

[3] L.G. Aslamazov and A.I. Larkin, Zh. Eksp. Teor. Fiz. 67, 647 (1974) [Sov. Phys. JETP 40, 321 (1975)].
[4] L.P. Lévy, D.H. Reich, L.N. Pfeiffer, and K.W. West, Physica (Amsterdam) 189B, 204 (1993).

[5] D. Mailly, C. Chapelier, and A. Benoit, Phys. Rev. Lett. 70, 2020 (1993).

[6] J.P. Eisenstein, H.L. Stormer, V. Narayanamurti, A.Y. Cho, A.C. Gossard, and C.W. Tu, Phys. Rev. Lett. 55, 875 (1985).

[7] A. Potts, R. Shepherd, W.G. Herrenden-Harker, M. Elliott, C.L. Jones, A. Usher, G.A.C. Jones, D.A. Ritchie, E.H. Linfield, and M. Grimshaw, J. Phys. Cond. Matt. 8, 5685 (1996).

[8] S.A.J. Wiegers, M. Specht, L.P. Lévy, M.Y. Simmons, D.A. Ritchie, A. Cavanna, B. Etienne, G. Martinez, and P. Wyder, Phys. Rev. Lett. 79, 3238 (1997).

[9] I. Meinel, T. Hengstmann, D. Grundler, D. Heitmann, W. Wegscheider, and M. Bichler, Phys. Rev. Lett. 82, 819 (1999).

[10] D. Ullmo, H.U. Baranger, K. Richter, F. von Oppen, and R.A. Jalabert, Phys. Rev. Lett. 80, 895 (1998).

[11] A.A. Abrikosov, L.P. Gorkov, and I.E. Dzyaloshinski, Methods of Quantum Field Theory in Statistical Physics (Prentice-Hall, Englewood Clifs, 1963).

[12] B.L Altshuler, A.G. Aronov, and A. Yu. Zyuzin, Sov. Phys.-JETP 57, 889 (1983).

[13] For a review see B.L. Altshuler and A.G. Aronov in Electron-electron interactions in Disordered systems, edited by A.L. Efros and M. Pollak (North-Holland, Amsterdam, 1985).

[14] V. Ambegaokar and U. Eckern, Phys. Rev. Lett. 65, 381 (1990).

[15] U. Eckern, Z. Phys. B 42, 389 (1991).

[16] M. Gutzwiller, Chaos in Classical and Quantum Mechanics (Springer, New York, 1990).

[17] A. Erdelyi, Higher Transcendental Functions, vol. II (McGraw-Hill, New York, 1955) section 10.15.

[18] Strictly speaking, the Bessel function approximation Eq. (31) and hence Eqs. (32) and (33) are valid only for large $n$. However, one checks by direct computation that in the present case, no serious errors are incurred when using Eq. (31) even for small $n$.

[19] B. Shapiro, Physica A 200, 498 (1992).

[20] F. von Oppen and E.K. Riedel, Phys. Rev. B 48, 9170 (1993); F. von Oppen, Phys. Rev. B 50, 17151 (1994).

[21] D. Ullmo, K. Richter, and R.A. Jalabert, Phys. Rev. Lett. 74, 383 (1995); K. Richter, D. Ullmo and R.A. Jalabert, Phys. Rep. 276, 1 (1996).

[22] D. Ullmo, H.U. Baranger, K. Richter, F. von Oppen, and R.A. Jalabert, unpublished.

[23] L.P. Lévy, G. Dolan, J. Dunsmuir, and H. Bouchiat, Phys. Rev. Lett. 64, 2074 (1990).

[24] V. Chandrasekhar, R.A. Webb, M.J. Brady, M.B. Ketchen, W.J. Gallagher, and A. Kleinsasser, Phys. Rev. Lett. 67, 3578 (1991).

[25] P. Mohanty, E.M.Q. Jariwala, M.B. Ketchen, and R.A. Webb, in Quantum Coherence and Decoherence, edited by K. Fujikawa and Y.A. Ono (Elsevier, 1996).

[26] For recent reviews see, e.g. U. Eckern and P. Schwab, Adv. Phys. 44, 387 (1995); K. Efetov, Supersymmetry in Disorder and Chaos (Cambridge University Press, 1996). 


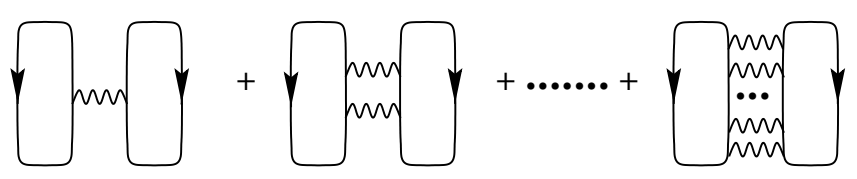

FIG. 1. Leading Cooper-channel diagrams for the interaction contribution to the thermodynamic potential. Because we can take the interaction to be local (a $\delta$-function), the corresponding Fock-like diagrams differ from the Hartree-like diagrams shown only by a factor of $-1 / 2$.
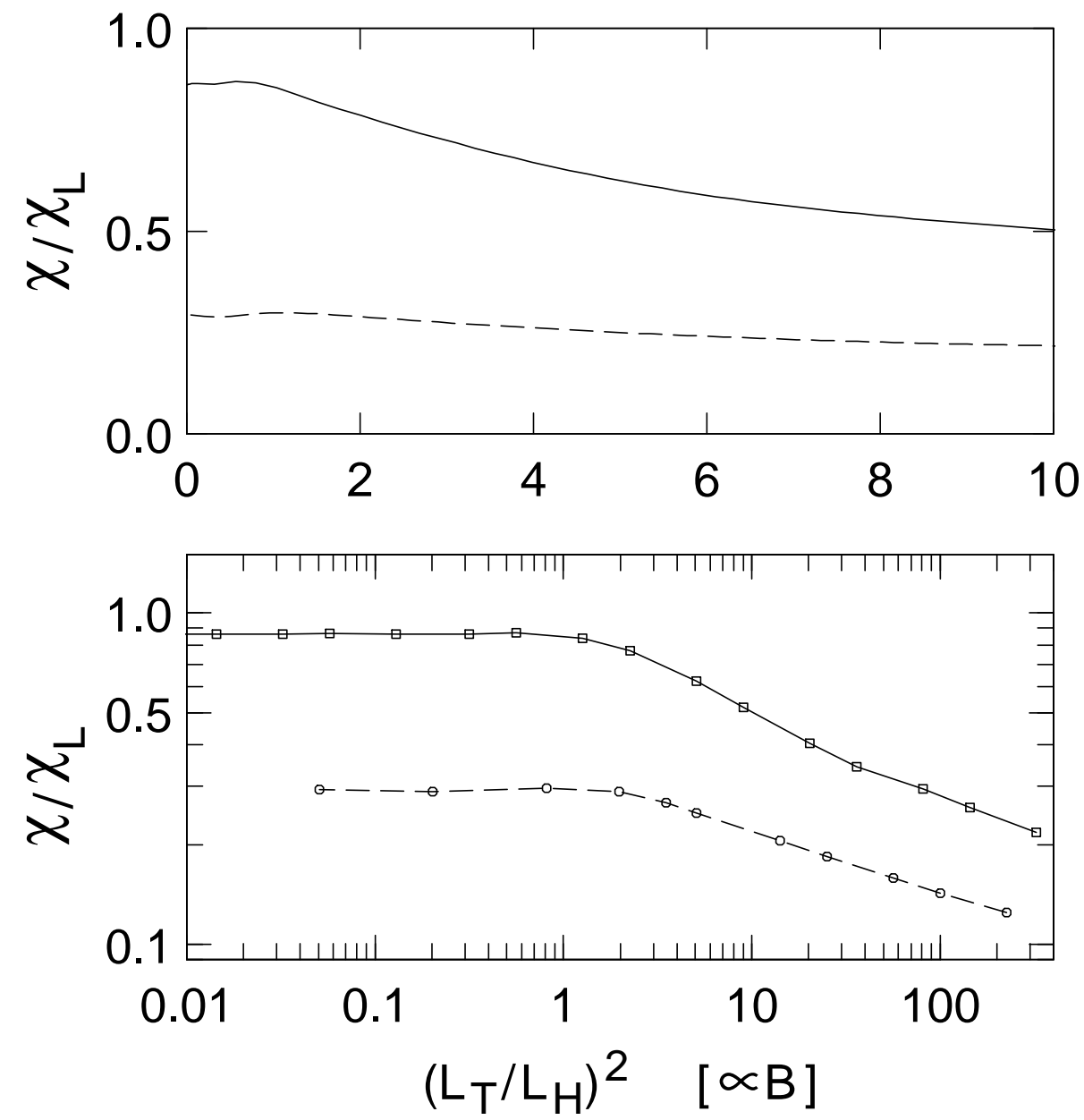

FIG. 2. Magnetic field dependence of the susceptibility at two temperatures, $E_{F} / T=2000$ (solid) and 200 (dashed). The Landau susceptibility $\chi_{L}$ is the natural unit for $\chi$; the lower panel shows the same data on an logarythmic scale. Note the cross-over in behavior when $L_{T} \approx L_{H}$. 


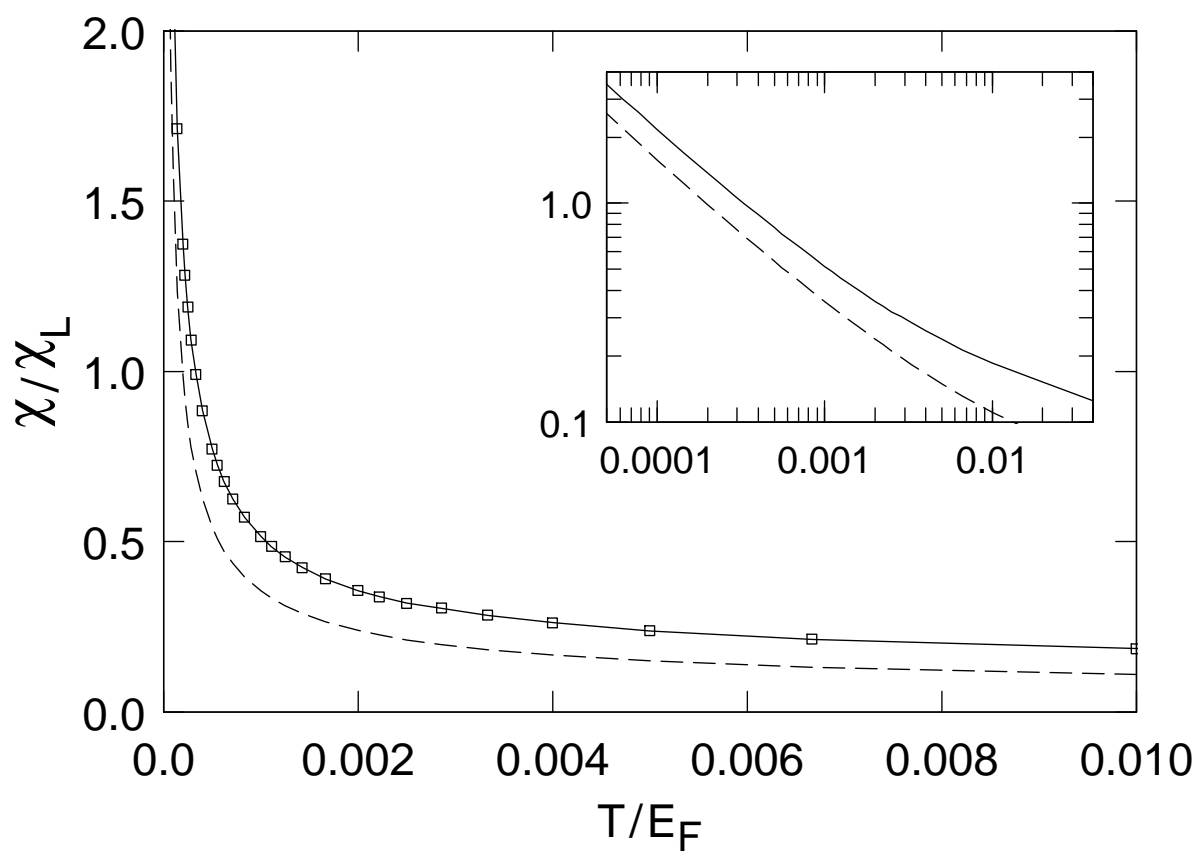

FIG. 3. Temperature dependence of the interaction-induced low-field susceptibility; $L_{H} / L_{T}=8.9$ is fixed. The total susceptibility differs from the interaction-induced contribution shown here only by a constant offset due to the Landau and Pauli contributions. The inset shows the same data on a log-log scale. For this low field, the contribution of the $\omega=0$ Matsubara frequency (dashed) gives a substantial portion of the result (solid). Note the approximate power law increase in $\chi$ at low temperatures, consistent with the asymptotic expression $1 / T \ln ^{3} T$.

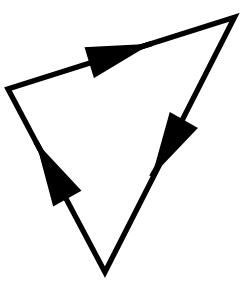

(a)

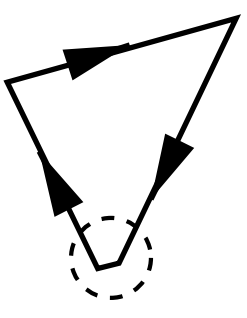

(b)

FIG. 4. (a) Typical trajectory of lowest order in the coupling constant which contributes to the interaction contribution to the magnetization. Each vertex of the polygon corresponds to an interaction event. Note that at least three interaction events are required to obtain a trajectory enclosing magnetic flux. This explains the fact that the interaction contribution to the magnetization is third-order in the (renormalized) interaction. (b) Higher-order contributions predominantly lead to a renormalization of the third-order result due to short trajectories like the one shown. 